\begin{tabular}{|c|l|}
\hline Title & Quantum lithography under imperfect conditions : effects of loss and dephasing on two-photon interference fringes \\
\hline Author(s) & Fujiwara, Hideki; Kawabe, Yoshio; Okamoto, Ryo; Takeuchi, Shigeki; Sasaki, Keiji \\
\hline Citation & $\begin{array}{l}\text { Journal of the Optical Society of A merica B : Optical Physics, 28(3), 422-431 } \\
\text { https:/doi.org/40.1364/JOSAB.28.000422 }\end{array}$ \\
\hline Issue Date & 2011-03-01 \\
\hline Doc URL & http://hdl.handle.net/2115/45032 \\
\hline Rights & ○ 2011 Optical Society of A merica \\
\hline Type & article \\
\hline File Information & JOSAB28_3_422-431.pdf \\
\hline
\end{tabular}

Instructions for use 


\title{
Quantum lithography under imperfect conditions: effects of loss and dephasing on two-photon interference fringes
}

\author{
Hideki Fujiwara, ${ }^{1,3}$ Yoshio Kawabe, ${ }^{1}$ Ryo Okamoto, ${ }^{1,2,3}$ Shigeki Takeuchi, ${ }^{1,2,3}$ and Keiji Sasaki ${ }^{1,3, *}$ \\ ${ }^{1}$ Research Institute for Electronic Science, Hokkaido University, Kita-20, Nishi-10, Kita-ku, \\ Sapporo, Hokkaido 001-0020 Japan \\ ${ }^{2}$ The Institute of Scientific and Industrial Research, Osaka University, Mihogaoka 8-1, Ibaraki, Osaka 567-0047, Japan \\ ${ }^{3}$ CREST, Japan Science and Technology Agency (JST), 4-1-8, Honcho, Kawaguchi-shi, Saitama 332-0012 Japan \\ *Corresponding author: sasaki@es.hokudai.ac.jp
}

Received June 17, 2010; revised December 10, 2010; accepted December 16, 2010; posted December 21, 2010 (Doc. ID 130126); published February 11, 2011

\begin{abstract}
We propose a method to simulate a two-photon interference fringe by using a density matrix in order to describe various types of imperfections in the input state. Using this method, we numerically discuss the influence of various imperfections in an input state, such as dephasing and misalignment, on the quality (visibility and period) of the two-photon interference fringes. Applying this method to experimental data, we succeeded in numerically reproducing a two-photon interference fringe using the experimentally obtained density matrix, in which almost no free fitting parameters are required. From the results, because the main cause of the degradation of an interference fringe was found to be the limited aperture size of a two-photon detector, we can observe a two-photon interference fringe with a visibility of up to $94 \%$ in the experiments if an efficient two-photon absorbing material or a two-photon detector with a sufficiently high spatial resolution can be used. @ 2011 Optical Society of America OCIS codes: $\quad 270.5585,270.5290$.
\end{abstract}

\section{INTRODUCTION}

Optical lithography has been widely used in the mass production of submicrometer-level structures, such as semiconductor devices. However, there exists a diffraction limit, called the Rayleigh limit: the minimum period of the interference fringe can never be smaller than $\lambda / 2$. Quantum lithography [1-3] is one interesting method for overcoming this limit. The literature proposed that by using a superposition of two states, " $N$-photon Fock state in path A and no photons in path B" and vice versa (NOON state), $N$-photon interference fringes having a minimum period of $\lambda /(2 N)$ can be realized. Even though there are various two-photon, three-photon, and four-photon experiments, all of them include changing the path difference of the interferometers [4-14].

Recently, we have successfully observed a two-photon interference fringe beating the diffraction limit [15]. In this experiment, a superposition of two-photon polarization states $\left(\frac{1}{\sqrt{2}}\{|2 H\rangle+|2 V\rangle\}\right)$ was generated from two beta barium borate (BBO) crystals [16] and converted into a path-entangled two-photon NOON state $\left(\frac{1}{\sqrt{2}}\{|2,0\rangle+|0,2\rangle\}\right)[\underline{17}, \underline{18}]$. Then, the two-photon path-entangled state was incident to the optical setup for stable interference [19], and a two-photon interference fringe was generated at the focal plane of a lens having a high numerical aperture. By scanning a high-spatial-resolution two-photon detector by using the probe of a near-field scanning optical microscope (NSOM), we succeeded in the observation of a two-photon interference fringe beating the diffraction limit.

During the experiment, we found that the quality of the input two-photon entangled state strongly affected the pattern and visibility of the two-photon interference fringe. However, the influence of various imperfections (e.g., loss and dephasing) of the input entangled photon source on the observed two-photon interference fringe pattern has not been clearly understood. Although several investigations on the relationship between the two-photon interference fringe and input state have been reported thus far [2,20,21], influences such as the mixing of another separable state (noise) and the nonorthogonality of the entangled photon polarizations, which can only be described by a density matrix, have not been discussed.

In this paper, we propose a method to numerically reconstruct two-photon interference fringe patterns from the arbitrary density matrix $(\rho)$ of the input two-photon state; the model is based on our previous experimental setup [15]. To clarify the influences of various imperfections of the input state on the interference fringe, we discuss, from the calculated results, the quality of the input state required for the observation of a reduced period having sufficiently high visibility, by taking into account specific imperfections such as the Werner [22] and nonmaximally entangled states [21]. Furthermore, we also discuss the cause of degradation of the visibility in the experimentally observed two-photon interference fringe by comparing the calculated results using the density matrix obtained from the experiment in Ref. [15]. We find that the observed fringe can be well reconstructed from the experimentally obtained density matrix of the two-photon source when the limited numerical aperture size of the NSOM tip is taken into account. This infers that the main sources of the degradation in the visibility were the resolution of our twophoton detector and the imperfections of the two-photon source. This knowledge will be important for not only the quantum lithography but also for further understanding of the "entangled state of photons."

This paper is organized as follows. In Section 2 , we introduce a method to simulate two-photon interference fringes from an arbitrary density matrix using the proposed setup. 
In Section 3 , by applying this method to several input state conditions, we discuss the quality of the input state required for realizing the subwavelength two-photon interference fringe with high visibility. In Section 4 , by experimentally measuring the density matrix of the input polarization entangled state and the interference fringe by using the setup used in Ref. [15], we confirm the relationship between the qualities of the input state and the fringe. Finally, we present our conclusions in Section $\underline{5}$.

\section{TWO-PHOTON INTERFERENCE FRINGE USING ARBITRARY DENSITY MATRIX}

\section{A. Two-Photon Interference Fringe with Ideal Input State}

Figure 1 shows a numerical model used in the following analysis, which schematizes the experimental setup proposed in Ref. [15]. In this model, a photon source generates a polarization entangled state in which two nonlinear crystals with orthogonal crystal axes satisfy the Type I collinear phasematching condition [16] and are excited by a diagonally polarized pump beam. The generated ideal input state is given by

$$
|\psi\rangle=\frac{1}{\sqrt{2}}\left\{|2 V\rangle_{s}+\exp (i \xi)|2 H\rangle_{s}\right\},
$$

where $\xi$ indicates the relative phase between the photon pairs generated from the different crystals. The subscript $s$ indicates that the photons are in path $s$. A calcite crystal spatially separates the generated entangled photon pairs into paths $a$ and $b$ depending on the polarization [19]. Then, a polarization aligner aligns the polarizations of the photons in paths $a$ and $b$ in which the polarization in one path is rotated by $90^{\circ}$, and the polarization entangled state is converted to the two-photon NOON state. The state in paths $c$ and $d$ is obtained as

$$
|\psi\rangle=\frac{1}{\sqrt{2}}\left\{|2 H\rangle_{c}|0\rangle_{d}+\exp (i \xi)|0\rangle_{c}|2 H\rangle_{d}\right\}
$$

This state is reflected to the paths $e$ and $f$ by mirrors and it interferes at the detection plane with the intersecting angle of $2 \theta$. Using this intersecting angle, the phase difference $\phi$ between the paths $e$ and $f$ can be transformed to the position vector $r$ on the detection plane as

$$
\phi=2 k r \sin \theta .
$$

Here, $r$ is the amplitude of the position vector $r$ on the detection plane. $k$ is the amplitude of the wave vector; $k=\left|\boldsymbol{k}_{e}\right|=$ $\left|\boldsymbol{k}_{f}\right|$, where $\boldsymbol{k}_{e}$ and $\boldsymbol{k}_{f}$ indicate the wave vectors in the paths $e$ and $f$, respectively, because we assume the interference of the monochromatic plane wave. Using the ideal polarization entangled state Eq. (1) as the input state, the two-photon detection efficiency $P(\boldsymbol{r})$ on the detection plane is given by [1]

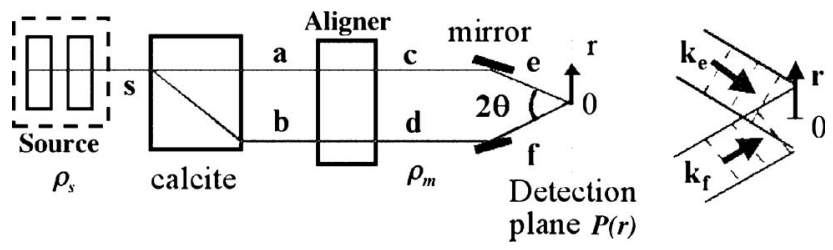

Fig. 1. Numerical model to simulate two-photon interference pattern.

$$
P(\boldsymbol{r})=\frac{1}{2}(1+\cos 2 \phi)=\frac{1}{2}\{1+\cos (4 k r \sin \theta)\},
$$

where $\xi=0$ is assumed. Thus, this equation shows that the fringe period reduced by a factor of $1 / 2$ can be obtained on the detection plane when a NOON state and two-photon detection are used.

\section{B. Two-Photon Interference Fringe with a Nonideal Input State}

The ideal input state, such as Eqs. (1) and (2), is difficult to obtain in actual experiments because of dephasing and misalignment of optical components. To treat such imperfections of the states, we use the density matrices of the two-photon polarization entangled state immediately after the photon source, $\rho_{s}$, and the two-photon path-entangled state after the polarization aligner and mirrors, $\rho_{m}$, and we consider the relationships between the density matrices $\left(\rho_{s}\right.$ and $\left.\rho_{m}\right)$ and the two-photon detection efficiency $(P(\boldsymbol{r}))$.

In order to study the influence of the input state quality on a two-photon interference fringe, it is necessary to obtain the relationship between $\rho_{s}$ and $\rho_{m}$. While the polarization entangled state generated from the two nonlinear crystals $\rho_{s}$ is described by the two-photon polarization basis, $\rho_{m}$ is described by the photon number basis, as mentioned above. As we assume a monochromatic plane wave in a single-mode propagation, a $3 \times 3$ matrix of the polarization basis is used owing to the indistinguishability between the states $|H V\rangle$ and $|V H\rangle$.

$$
\begin{aligned}
\rho_{s}= & b_{11}|2 V\rangle_{s}\left\langle\left. 2 V\right|_{s}+b_{12} \mid 2 V\right\rangle_{s}\left\langle\left. V H\right|_{s}+b_{13} \mid 2 V\right\rangle_{s}\left\langle\left. 2 H\right|_{s}\right. \\
& +b_{12}^{*}|V H\rangle_{s}\left\langle\left. 2 V\right|_{s}+b_{22} \mid V H\right\rangle_{s}\left\langle\left. V H\right|_{s}+b_{23} \mid V H\right\rangle_{s}\left\langle\left. 2 H\right|_{s}\right. \\
& +b_{13}^{*}|2 H\rangle_{s}\left\langle\left. 2 V\right|_{s}+b_{23}^{*} \mid 2 H\right\rangle_{s}\left\langle\left. V H\right|_{s}\right. \\
& +\left(1-b_{11}-b_{22}\right)|2 H\rangle_{s}\left\langle\left. 2 H\right|_{s},\right.
\end{aligned}
$$

where $b_{i j}(i, j=1,2,3)$ indicates the complex coefficients for the density matrix $\rho_{s}$.

In order to discuss the relationship between $\rho_{s}$ and $\rho_{m}$, the influences of the calcite crystal, the polarization aligner, and the two-photon detector should be considered. Ignoring the misalignment of the optical axis of the calcite crystal against the polarizations of the photons, the state of the photons in paths $a$ and $b$ can be obtained by simply replacing the subscript $s$ with $a$ or $b$ depending on the polarization in Eq. (5) $\left(|2 H\rangle_{s} \rightarrow|0\rangle_{a}|2 H\rangle_{b}\right.$ and $\left.|2 V\rangle_{s} \rightarrow|2 V\rangle_{a}|0\rangle_{b}\right)$. Then, because we used a polarization aligner for aligning the polarizations of the photons in paths $a$ and $b$, we transform the basis from $H$ to $V$ or from $V$ to $H$. In addition, because we pay attention to the two-photon interference, only the terms including the twophoton state $(|2 V\rangle,|V\rangle|V\rangle,|2 H\rangle$, and $|H\rangle|H\rangle)$ remain, resulting in the basis transformation of $|2 H\rangle_{s} \rightarrow|0\rangle_{e}|2\rangle_{f},|2 V\rangle_{s} \rightarrow$ $|2\rangle_{e}|0\rangle_{f}$, and $|H V\rangle_{s} \rightarrow|1\rangle_{e}|1\rangle_{f}$. As a result, using the coefficients of $\rho_{s}, \rho_{m}$ is described as follows: 


$$
\begin{aligned}
\rho_{m} & =b_{11}|2\rangle_{e}|0\rangle_{f}\left\langle\left. 0\right|_{f}\left\langle\left. 2\right|_{e}+b_{12} \mid 2\right\rangle_{e} \mid 0\right\rangle_{f}\left\langle1 | _ { f } \left\langle\left. 1\right|_{e}\right.\right. \\
& +b_{13}|2\rangle_{e}|0\rangle_{f}\left\langle\left. 2\right|_{f}\left\langle\left. 0\right|_{e}+b_{12}^{*} \mid 1\right\rangle_{e} \mid 1\right\rangle_{f}\left\langle0 | _ { f } \left\langle\left. 2\right|_{e}\right.\right. \\
& +b_{22}|1\rangle_{e}|1\rangle_{f}\left\langle\left. 1\right|_{f}\left\langle\left. 1\right|_{e}+b_{23} \mid 1\right\rangle_{e} \mid 1\right\rangle_{f}\left\langle2 | _ { f } \left\langle\left. 0\right|_{e}\right.\right. \\
& +b_{13}^{*}|0\rangle_{e}|2\rangle_{f}\left\langle\left. 0\right|_{f}\left\langle\left. 2\right|_{e}+b_{23}^{*} \mid 0\right\rangle_{e} \mid 2\right\rangle_{f}\left\langle1 | _ { f } \left\langle\left. 1\right|_{e}\right.\right. \\
& +\left(1-b_{11}-b_{22}\right)|0\rangle_{e}|2\rangle_{f}\left\langle2 | _ { f } \left\langle\left. 0\right|_{e} .\right.\right.
\end{aligned}
$$

In this equation, we omit the polarization notation because the polarizations in each path are the same owing to the polarization aligner.

Using $\rho_{m}$, we can simulate the two-photon interference fringe on the detection plane from the calculation of the two-photon detection efficiency, $P(\boldsymbol{r})$ :

$$
P(\boldsymbol{r})=\operatorname{Tr}\left\{\rho_{m} \hat{E}^{(-)}(\boldsymbol{r}) \hat{E}^{(-)}(\boldsymbol{r}) \hat{E}^{(+)}(\boldsymbol{r}) \hat{E}^{(+)}(\boldsymbol{r})\right\},
$$

where $\hat{E}^{(+)}(\boldsymbol{r})\left(\hat{E}^{(-)}(\boldsymbol{r})\right)$ indicates the positive (negative) frequency component of the electric field operator and is described by the superposition of the plane waves propagating in paths $e$ and $f$ :

$$
\begin{aligned}
& \hat{E}^{(+)}(\boldsymbol{r})=\frac{1}{\sqrt{2}}\left\{\hat{a}_{e} \exp \left(i \boldsymbol{k}_{e} \cdot \boldsymbol{r}\right)+\hat{a}_{f} \exp \left(i \boldsymbol{k}_{f} \cdot \boldsymbol{r}\right)\right\}, \\
& \hat{E}^{(-)}(\boldsymbol{r})=\left\{\hat{E}^{(+)}(\boldsymbol{r})\right\}^{\dagger},
\end{aligned}
$$

where $\hat{a}_{e}, \hat{a}_{f}, \boldsymbol{k}_{e}$, and $\boldsymbol{k}_{f}$ are the annihilation operators and the wave vectors of the photons propagating in paths $e$ and $f$. Substituting Eqs. (6) and (8) into Eq. (7), the two-photon detection probability as a function of $\phi$ can be given by

$$
\begin{aligned}
P(\phi)= & \frac{1}{2}\left[1+b_{22}+\left(b_{13}+b_{13}^{*}\right) \cos 2 \phi+i\left(b_{13}-b_{13}^{*}\right) \sin 2 \phi\right. \\
& +\sqrt{2}\left(b_{12}+b_{23}+b_{12}^{*}+b_{23}^{*}\right) \cos \phi \\
& \left.+\sqrt{2} i\left(b_{12}+b_{23}-b_{12}^{*}-b_{23}^{*}\right) \sin \phi\right] .
\end{aligned}
$$

Here $\phi$ is the phase difference given by Eq. (3). Using Eq. (3), we obtain the two-photon detection efficiency on the detection plane with the position vector $\boldsymbol{r}$ as

$$
\begin{aligned}
P(\boldsymbol{r})= & \frac{1}{2}\left[1+b_{22}+\left(b_{13}+b_{13}^{*}\right) \cos (4 k r \sin \theta)\right. \\
& +i\left(b_{13}-b_{13}^{*}\right) \sin (4 k r \sin \theta) \\
& +\sqrt{2}\left(b_{12}+b_{23}+b_{12}^{*}+b_{23}^{*}\right) \cos (2 k r \sin \theta) \\
& \left.+\sqrt{2} i\left(b_{12}+b_{23}-b_{12}^{*}-b_{23}^{*}\right) \sin (2 k r \sin \theta)\right],
\end{aligned}
$$

where $r$ indicates the amplitude of $\boldsymbol{r}$.

\section{Effect of Intensity Distribution on Two-Photon Interference Fringe}

Although the above discussion assumes plane-wave propagation, the spatial distribution of the photon pairs typically has a Gaussian profile depending on the mode profile of the pump beam $\left(\mathrm{TEM}_{00}\right)$. In order to incorporate the influence of the beam profile, we simply multiply Eq. (10) by the Gaussian function. When the variance, center position, and amplitude of the Gaussian function were set as $\sigma^{2}, r_{0}$, and $a$, respectively, the interference fringe on the detection plane in Eq. (9) and (10) is weighted by the Gaussian function and is given as

$$
P_{\text {gauss }}(\boldsymbol{r})=a \exp \left\{-\frac{\left(r-r_{0}\right)^{2}}{2 \sigma^{2}}\right\} \times P(\boldsymbol{r})
$$

If we use an efficient two-photon absorbing material having a sufficiently high spatial resolution, we would observe the interference fringe $P_{\text {gauss }}(\boldsymbol{r})$ on the detection plane in the experiments.

\section{Effect of the Limited Aperture Size of the Detector}

In our previous experiment [15], we used a high-resolution two-photon detector, such as the probe of a near-field scanning microscope (NSOM) connected to two single-photon counting modules. Because the limited aperture size of an NSOM probe was not negligibly smaller than the period of the two-photon interference fringe, the influence of the aperture on the fringe cannot be ignored. To account for its effect, the calculated two-photon interference fringe weighted by the Gaussian function [Eq. (11)] is convolved with a rectangular function with the width of the average aperture size $d$. Therefore, the two-photon interference fringe observed by the twophoton detector with limited aperture size is given as

$$
P_{\mathrm{obs}}(\boldsymbol{r})=\int_{0}^{r} \operatorname{rect}(x / d) P_{\text {gauss }}(r-x) \mathrm{d} x,
$$

where

$$
\operatorname{rect}(x / d)=\left\{\begin{array}{cc}
0 & \text { if }|x|>d / 2 \\
1 & \text { otherwise }
\end{array},\right.
$$

where $\operatorname{rect}(x / d)$ is the rectangular function and $d$ is the aperture size of the NSOM probe. Using this equation, we can simulate the two-photon interference fringe on the detection plane observed by the NSOM probe, which can be compared with the experimental data [15]. Note that most of the parameters (density matrix, beam width, and aperture size) can be determined experimentally, and only a few fitting parameters (such as the unknown bias phase for the interference fringe in an interferometer) were required for the calculation.

\section{INFLUENCE OF QUALITY OF ENTANGLED PHOTON SOURCE ON FRINGE QUALITY}

In this section, we discuss the influence of the input polarization entangled state on the two-photon interference fringe pattern when $\rho_{s}$ dissociates from the ideal polarization entangled state represented in Eq. (1). As seen in Eq. (9), the fringe quality is easily degraded according to the imperfection of the input state. Here we consider several specific cases for the degradation of the fringe quality, such as relative phase shift, mixing of another separable state, unbalanced coupling between $|2 H\rangle$ and $|2 V\rangle$ (nonmaximal entanglement), and nonorthogonality between linearly polarized two-photon states. For this purpose, using Eq. (9), two-photon interference fringes are simulated from the following input states: (i) ideal polarization entangled state with a different relative phase shift, (ii) Werner state, (iii) nonmaximally entangled state, and (iv) superposition of the nonorthogonal linearly polarized two-photon states. 


\section{A. Polarization Entangled State with Different Relative Phase}

First, we discuss the influence of the relative phase change $\xi$ between $|2 H\rangle$ and $|2 V\rangle$ in Eq. (1) on the fringe quality. Figure 2 represents the two-photon detection efficiencies calculated from the polarization entangled state by changing the relative phase $\xi$ from 0 to $\pi$. The data were normalized by each maximum value. Even when we can prepare the ideal polarization entangled state, the two-photon interference fringes with the visibility of 1 and with the reduced period of $\pi$ are shifted depending on the relative phase. This result suggests that the fluctuations of the phase difference, e.g., those due to the instability of the interferometer, easily wash out the fringe because a long accumulation time is typically required in quantum interference experiments. Thus, not only the highquality entangled photon source but also the stable interferometer is indispensable, while the fringe visibility and period are not susceptible to the change of the relative phase.

\section{B. Werner State}

In order to discuss the influence of the mixing of other states in the input state, we consider the Werner state as the input state [22]. The density matrix of the Werner state, which is given by the mixture of a maximally entangled state and a statistical mixture of $|2 H\rangle,|2 V\rangle$, and $|H V\rangle$ with equal probabilities (a unit matrix state $I^{(3)}$ ), is

$$
\rho_{w}=t|\psi\rangle\langle\psi|+\frac{1-t}{3} I^{(3)},
$$

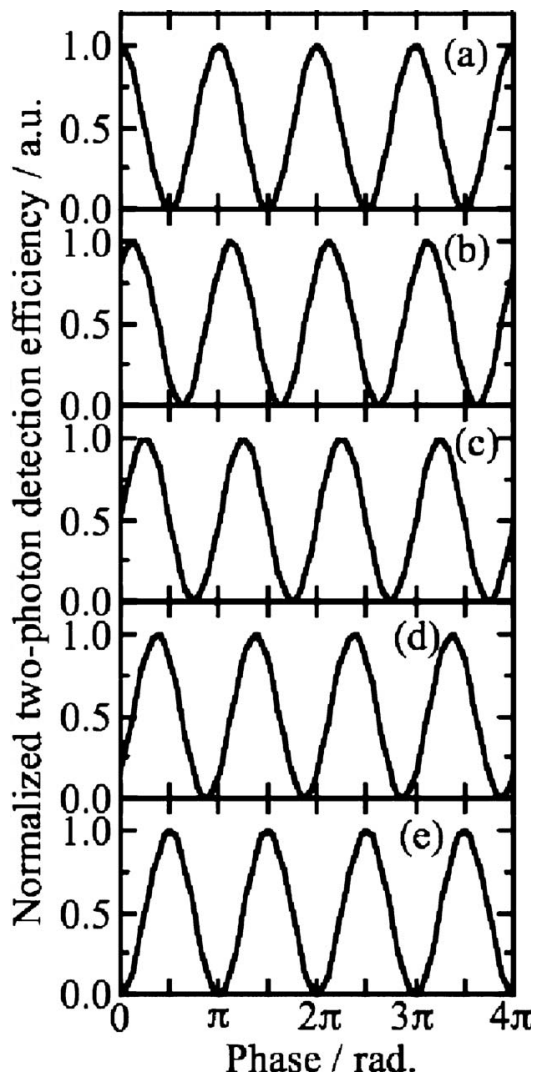

Fig. 2. Calculated interference fringes using maximally entangled state by changing the relative phase $\xi$ from 0 (top) to $\pi$ (bottom) in Eq. (1): (a) $\xi=0$, (b) $\xi=\pi / 4$, (c) $\xi=\pi / 2$, (d) $\xi=3 \pi / 4$, and (e) $\xi=\pi$. where $t$ indicates the fraction of the polarization entangled state. In this state, the coefficients of the density matrix are $b_{11}=(2+t) / 6, b_{12}=b_{23}=0, b_{13}=t / 2$ and $b_{22}=(1-t) / 3$ (see right side in Fig. 3). Using this input state, the obtained two-photon detection efficiency is given by

$$
P(\phi)=\frac{1}{2}\left(\frac{1-t}{3}+1+t \cos 2 \phi\right) .
$$

The visibility is equal to $3 t /(4-t)$. From Eq. (14), the fraction of the bias component $[(1-t) / 3]$ against the fringe component $(1+t \cos 2 \phi)$ increases with the decrease of the fraction $t$ of the entangled state in the input state.

Figure 3 represents the calculated two-photon detection efficiency obtained by changing the fraction $t$ of the polarization entangled state in the Werner states. The data were normalized by the maximum value of the fringe with the ideal input state $(t=1)$. In addition, the calculated density matrices for different $t$ [(a) 1 , (c) 0.5 , and (e) 0 ] are shown on the right side of Fig. 3. Depending on the fraction $t$, the visibility of the fringe decreases from $1(t=1)$ to $0(t=0)$, while maintaining the reduced period of $\pi$ constant. As mentioned above, this result can be intuitively understood from the definition of the Werner state [Eq. (13)]. In this equation, the first term $(1-t) / 3$, which is a fraction of the unit matrix, acts as the bias component in the interference pattern, while the other term $(1+t \cos 2 \phi)$, generates the interference fringe with a reduced period of $\pi$ and a visibility of $t$, which originates from the entangled state in the input state. This effect also appeared in the density matrices. When the fraction of the entangled state, $t$, was decreased, an increase in the $|H V\rangle\langle H V|$ component and decrease in the $|2 H\rangle\langle 2 V|$ and $|2 V\rangle\langle 2 H|$ components were observed, and only the $I^{(3)}$ component remained when
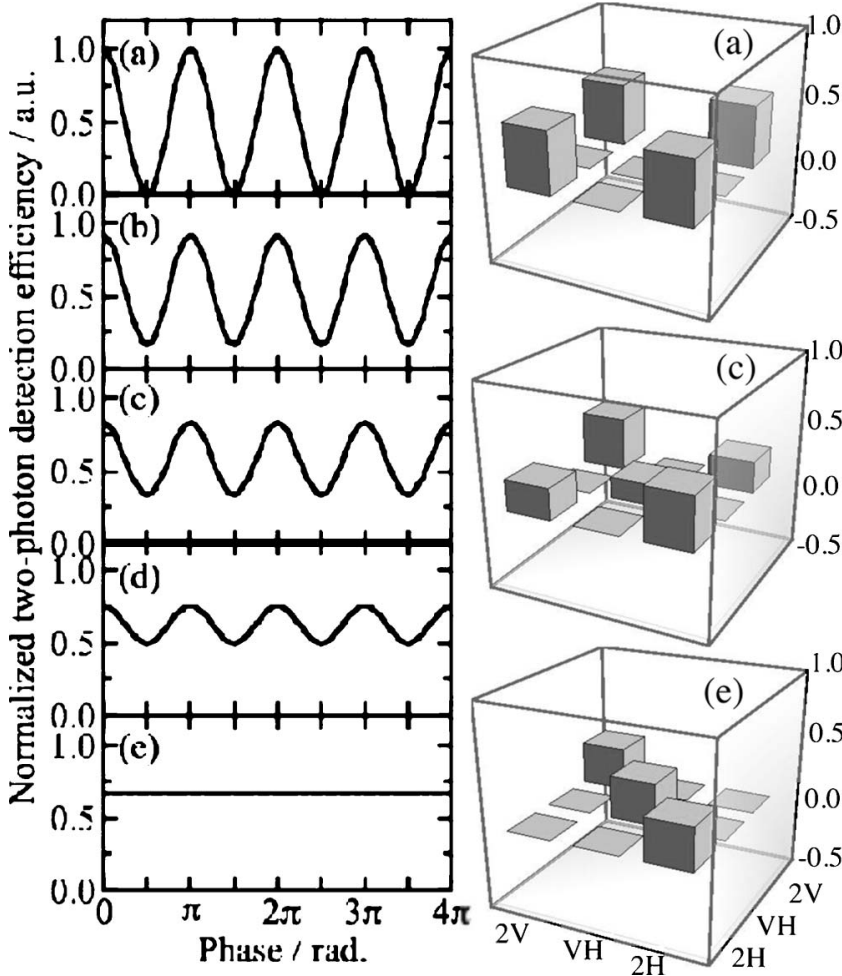

Fig. 3. Calculated interference fringes and density matrices using Werner state by changing the fraction $t$ from 0 (top) to 1 (bottom); (a) $t=1$, (b) $t=0.75$, (c) $t=0.5$, (d) $t=0.25$, and (e) $t=0$. 
$t=0$. This indicates that the entanglement of the input state decreased, resulting in the disappearance of the interference. Thus, this result suggests that the fraction of the entangled state must be high in order to realize a two-photon interference fringe with high visibility.

\section{Nonmaximally Entangled State}

From Eq. (9), even if the balance between the $|2 H\rangle$ and $|2 V\rangle$ components in the polarization entangled state is disrupted, the interference fringe quality is strongly modified. This unbalance may occur if the linear polarization of the pump beam is tilted from $45^{\circ}$. Here, as an example, we consider a nonmaximally entangled state [21], which is defined as

$$
|\psi\rangle=\cos \gamma|2 H\rangle+\sin \gamma|2 V\rangle,
$$

where $\gamma$ determines the ratio between $|2 H\rangle$ and $|2 V\rangle$. From this definition, the coefficients of the density matrix are determined as $b_{11}=\sin ^{2} \gamma, b_{12}=b_{22}=b_{23}=0$, and $b_{13}=$ $\cos \gamma \sin \gamma$, respectively. Substituting these coefficients into Eq. (9), the two-photon detection efficiency is given by

$$
P(\phi)=\frac{1}{2}(1+\sin 2 \gamma \cos 2 \phi),
$$

and the visibility is $\sin 2 \gamma$.

The calculated two-photon detection efficiencies and the calculated density matrices for different $\gamma\left(45^{\circ}, 30^{\circ}, 20^{\circ}\right.$, $10^{\circ}$, and $0^{\circ}$ ) are shown in Fig. 4. The calculated interference fringes were normalized by the maximum value of the fringe with the ideal input state $\left(\gamma=45^{\circ}\right)$. When $\gamma=45^{\circ}$, which corresponds to the maximally polarized entangled state [Fig. 4(a)], the visibility of the interference fringe with the per$\operatorname{iod} \pi$ is maximized to 1 . When decreasing $\gamma$ to $0^{\circ}$ or increasing to $90^{\circ}$, an unbalance between the $|2 H\rangle$ and $|2 \mathrm{~V}\rangle$ components in the density matrix is observed and the fringe visibility decreases while maintaining the reduced period $\pi$ constant [Fig. 4(c)]. When $\gamma$ is $0^{\circ}$ or $90^{\circ}$, the fringe disappears, which indicates that the input state is not entangled, but the photon number state $|2 H\rangle$ or $|2 V\rangle$ is clearly shown in the density matrix of Fig. 4(e). This result suggests that the fraction of the generated $|2 \mathrm{~L}\rangle$ and $|2 \mathrm{~V}\rangle$ components from the different crystals must be balanced to achieve the maximally entangled state for a fringe with high visibility.

\section{Effect of the Extra Two-Photon Polarization Component $|\boldsymbol{H V}\rangle$}

The increase in the $|H V\rangle$ component, because of the rotation of the polarization and the nonorthogonality of the optical axes between the two nonlinear crystals, also affects the fringe pattern. From Eq. (9), because the increase in the $|H V\rangle$ component results in the increase in the fringe component with a fundamental period of $2 \pi$, not only the visibility but also the fringe period change. Here, as an example of this specific case, we consider the influence of the nonorthogonality of the optical axes between two nonlinear crystals on the fringe quality. We assume a state $|2 x\rangle$ such that the angle between the polarizations of the $|2 H\rangle$ component and the diagonally polarized pump beam changes from $45^{\circ}$ by $x^{\circ}$, while the $45^{\circ}$ angle remains between the polarizations of the $|2 \mathrm{~V}\rangle$ component and the pump beam because of the misalignment of one crystal. Thus, the state of $|2 x\rangle$ includes both $H$ and $V$ com-
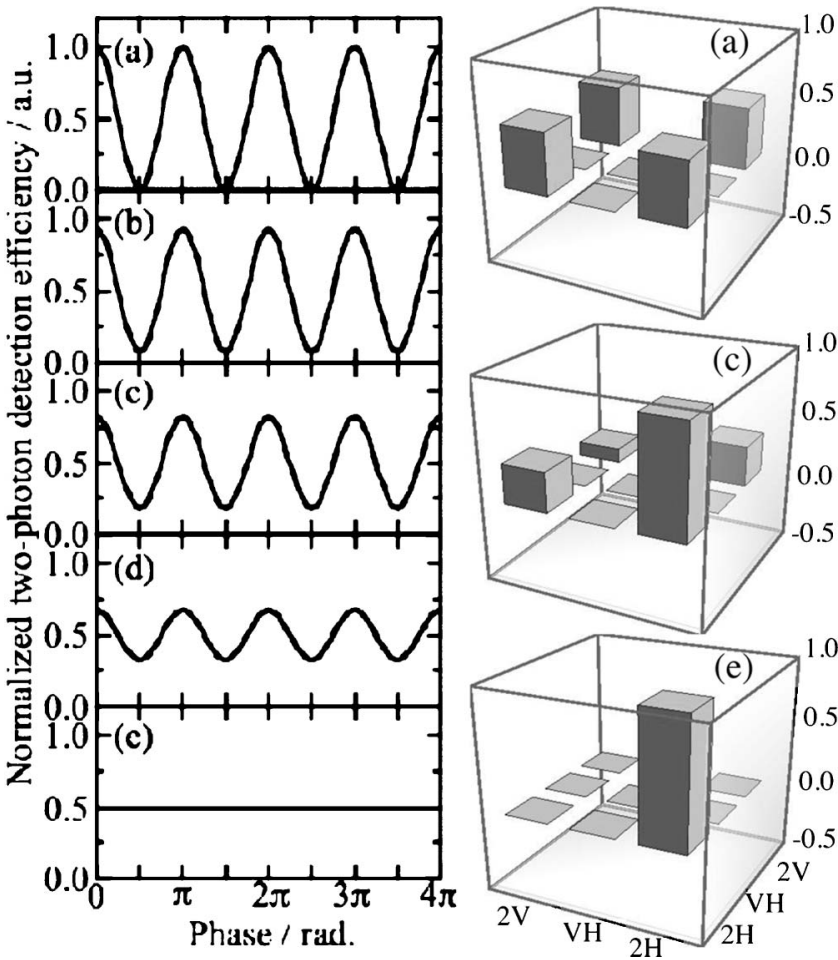

Fig. 4. Calculated interference fringes and density matrices using nonmaximally entangled states with changing $\gamma: \gamma=$ (a) $45^{\circ}$, (b) $30^{\circ}$, (c) $20^{\circ}$, (d) $10^{\circ}$, and (e) $0^{\circ}$.

ponents, resulting in the generation of the $|H V\rangle$ component. Because the state of $|2 x\rangle$ can be defined as

$$
\begin{aligned}
& |2 x\rangle=\frac{1}{\sqrt{2}} \hat{a}_{x}^{\dagger} \hat{a}_{x}^{\dagger}|0\rangle \\
& =\frac{1}{\sqrt{2}}\left(\hat{a}_{H}^{\dagger} \cos x+\hat{a}_{V}^{\dagger} \sin x\right)\left(\hat{a}_{H}^{\dagger} \cos x+\hat{a}_{V}^{\dagger} \sin x\right)|0\rangle,
\end{aligned}
$$

where $\hat{a}_{j}^{\dagger}(j=x, H, V)$ is the creation operator of a photon with $j$ polarization, the normalized input state is given by

$$
\begin{gathered}
|\psi\rangle=\frac{c}{\sqrt{2}}(|2 V\rangle+\alpha|2 x\rangle) \\
=\frac{c}{\sqrt{2}}\left(\alpha \cos ^{2} x|2 H\rangle+\sqrt{2} \alpha \cos x \sin x|H V\rangle\right. \\
\left.\quad+\left(1+\alpha \sin ^{2} x\right)|2 V\rangle\right),
\end{gathered}
$$

where

$$
\begin{gathered}
c=\sqrt{\frac{2}{1+2 \alpha \sin ^{2} x+\alpha^{2}}}, \\
\alpha=\sqrt{2}\left|\cos \left(x-\frac{\pi}{4}\right)\right| .
\end{gathered}
$$

In the above equations, $c$ is the normalized constant and $\alpha$ is the excitation efficiency of the $|2 x\rangle$ component using the diagonally polarized pump beam. Thus, as the coefficients of the density matrix are $b_{11}=c^{2}\left(1+\alpha \sin ^{2} x\right)^{2} / 2, b_{12}=$ $c^{2} \alpha \sin 2 x\left(1+\alpha \sin ^{2} x\right) / 2 \sqrt{2}, \quad b_{13}=c^{2} \alpha \cos ^{2} x\left(1+\alpha \sin ^{2} x\right) / 2$, $b_{22}=c^{2} \alpha^{2} \sin ^{2} 2 x / 4$, and $b_{23}=c^{2} \alpha^{2} \sin 2 x \cos ^{2} x / 2 \sqrt{2}$ from 
Eq. (18), the two-photon detection efficiency is described as

$$
\begin{aligned}
P(\phi)= & \frac{1}{2}\left\{1+\frac{c^{2} \alpha^{2} \sin ^{2} 2 x}{4}+c^{2} \alpha \sin 2 x(1+\alpha) \cos \phi\right. \\
& \left.+c^{2} \alpha \cos ^{2} x\left(1+\alpha \sin ^{2} x\right) \cos 2 \phi\right\} .
\end{aligned}
$$

Figure $\underline{5}$ shows the calculated two-photon detection efficiencies and the density matrices obtained by changing the degree of the misalignment from $-60^{\circ}$ to $60^{\circ}$ using Eq. (21). The interference fringes were normalized by the maximum value of the fringe with the ideal input state $\left(x=0^{\circ}\right)$. When $x=0^{\circ}$, where the crystals are orthogonally aligned and the ideal polarization entangled state is generated, a two-photon interference fringe with a high visibility of 1 is obtained, as shown in Fig. 5(c). However, by increasing or decreasing the tilting angle $x$ from $0^{\circ}$, the period of the two-photon interference fringe increases and the visibility worsens. Even when only a small misalignment occurs, i.e., less than $\pm 10^{\circ}$, the shape of the two-photon interference fringe begins to collapse and its profile is asymmetrically changed against the tilting angle $x$. With a further increase in the tilting angle, the visibility of the two-photon interference fringe with the fundamental period increases, while with a decrease of the tilting angle, the visibility of the two-photon interference fringe with the fundamental period decreases. At $x=45^{\circ}$, only the fundamental period of $2 \pi$ appears with sharpened fringe peaks because of the two-photon detection, while at $x=-45^{\circ}$, the interference fringe disappears. These behaviors can also be understood from the calculated density matrix. Compared with the density matrix and Eqs. (5) and (9), by increasing or decreasing the tilting angle $x$ from $0^{\circ}$, the $|H V\rangle$ component was increased and the balance between the $|2 H\rangle$ and $2 V\rangle$ compo- nents was also broken (increase in the $|2 V\rangle$ component). This indicates that the increase in $|2 V\rangle\langle H V|| H V\rangle,\langle 2 V|| 2 H\rangle,\langle H V|$, and $|H V\rangle\langle 2 H|$ components induces an increase in the interference fringe component with the fundamental period, and the increase in the $|H V\rangle\langle H V|$ component increases the bias component, resulting in decreased visibility. Thus, according to the change in the density matrix from the ideal state, the quality of the two-photon interference fringe rapidly worsens. Therefore, the fringe period and visibility are degraded by mixing a small amount of the $|H V\rangle$ component because of the rotation of the polarization and the nonorthogonality of the crystals.

As shown in the above subsections, we analyzed the influences of several specific input states on the quality of the twophoton interference fringe (visibility and period) by using our proposed method. From these results, we can summarize that the purification of the state (suppression of the mixing of another separable state), maximization of the entanglement, and suppression of the polarization rotation in the optics are indispensable for the realization of a two-photon interference fringe with a reduced period smaller than $\lambda / 2$ and sufficiently high visibility.

\section{EXPERIMENTAL VERIFICATION}

In this section, in order to verify the numerical results, we experimentally measured the density matrix of the input polarization entangled state and the two-photon interference fringe by using the same setup proposed in Ref. [15], and compared the result with the calculated result using experimental parameters.

Figure 6 shows a schematic of the experimental setup. In the experiment, the polarization entangled state was generated from two BBO crystals for the Type I collinear
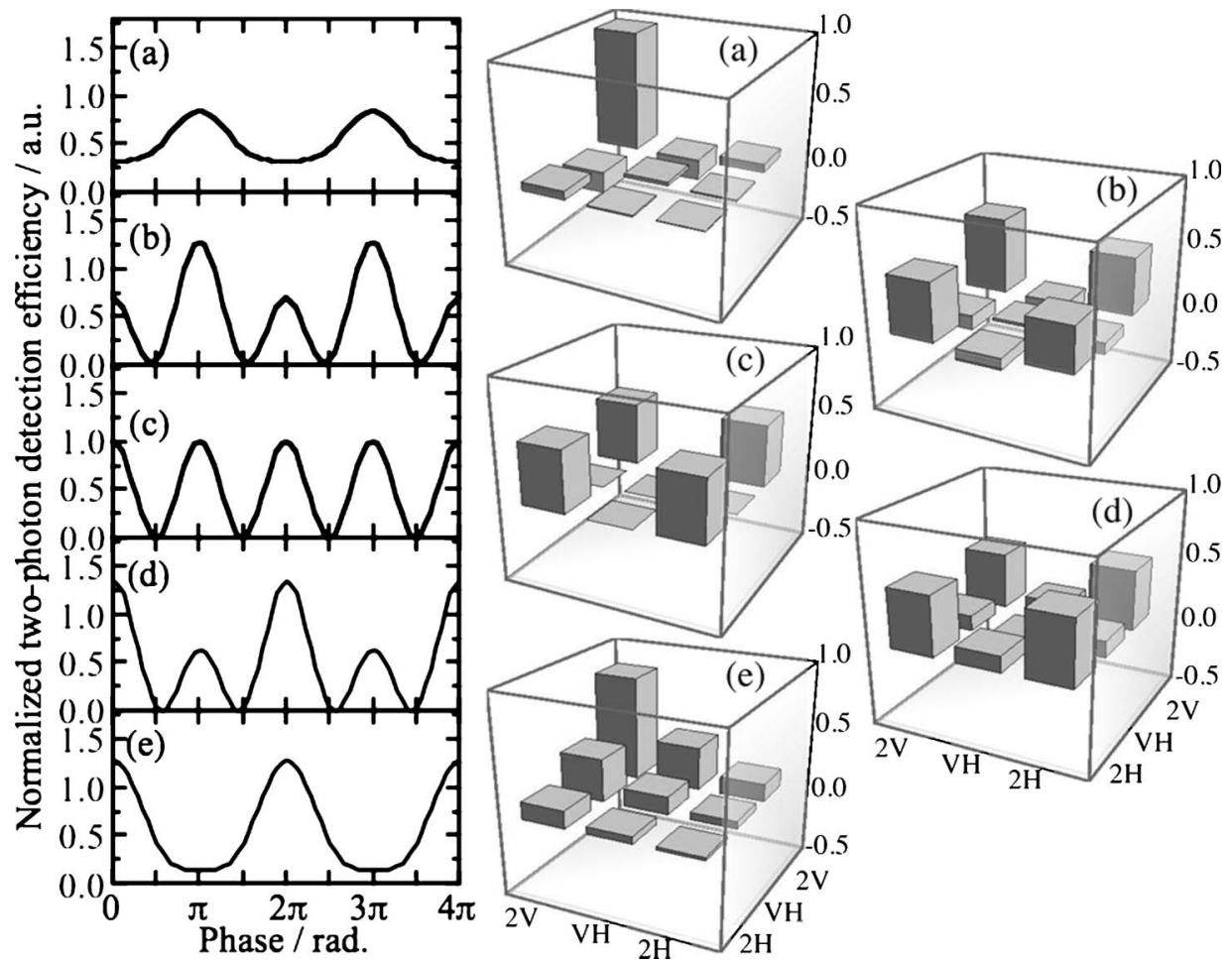

Fig. 5. Calculated interference fringes and density matrices using the superposition state of the nonorthogonal linearly polarized two-photon states by changing the tilting angle: $x=(\mathrm{a})-60^{\circ}$, (b) -10 , (c) $0^{\circ}$, (d) $10^{\circ}$, and (e) $60^{\circ}$. 
phase-matching condition, in which the optical axes of the crystals were set orthogonal to each other and also diagonal to the linearly polarized pump beam $\left(\mathrm{CW} \mathrm{Ar}^{+}\right.$laser, wavelength $351.1 \mathrm{~nm}$ ) [16]. The generated photon pairs were passed through an interference filter (center wavelength $702.2 \mathrm{~nm}$, full width at half maximum $10 \mathrm{~nm}$ ) and a singlemode fiber (SMF). After passing through the SMF, we compensated for the polarization rotation in the SMF by using a half-wave plate (HWP) and a quarter-wave plate in order to achieve the high fidelity of the polarization entangled state. The polarization entangled photons were spatially separated into two paths $a$ and $b$ depending on their polarization in a calcite crystal [19]. Inserting a HWP in one path, the polarization was rotated by $90^{\circ}$, giving a two-photon NOON state $\left(\left(|2\rangle_{c}|0\rangle_{d}+|0\rangle_{c}|2\rangle_{d}\right) / \sqrt{2}\right.$ ). An aspheric lens (clear aperture $=3.6 \mathrm{~mm}, \quad \mathrm{NA}=0.65$ ) was used to focus the photons to a small spot and form an interference fringe on the focal plane. Then, an NSOM probe with an elliptical opening $(1.8 \mu \mathrm{m} \times 0.2 \mu \mathrm{m}$; see inset in Fig. 6$)$ was scanned along the focal plane, which was used as a high-resolution spatial filter. The SMF output of the NSOM probe was divided by a $50 \%$ beam splitter (BS) and detected by two single-photon counting modules (SPCMs; SPCM-AQR14FC, PerkinElmer) [23]. The coincidence events between the two SPCMs were recorded by a gated photon counter (SR-400, Stanford Research Systems). By scanning the NSOM probe with a $25 \mathrm{~nm}$ step with an accumulation time of $5 \mathrm{~s}$ for one data point, we measured the two-photon interference fringes.

In order to experimentally obtain the density matrix of the incident two-photon state, we performed quantum state tomography [24] of the polarization entangled photon source by using the setup shown in Fig. 7. Following Ref. [24], we measured the coincidence counts of projection measurements $(|\psi\rangle\langle\psi|)$ for a set of 16 data points that allowed the density matrix of the state of the two modes to be estimated. We used the notations $|\psi\rangle=|H\rangle,|V\rangle,|R\rangle,|L\rangle$, and $|D\rangle$, where $|D\rangle=$ $(|H\rangle+|V\rangle) / \sqrt{2}, \quad|R\rangle=(|H\rangle-i|V\rangle) / \sqrt{2} \quad$ and $\quad|L\rangle=(|H\rangle+$ $i|V\rangle) / \sqrt{2}$. From the measurements, we determined the coefficients of the $4 \times 4$ density matrix $\rho_{s}$. Then, the obtained $\rho_{s}$ was converted into a $3 \times 3$ matrix assuming that the two-photon

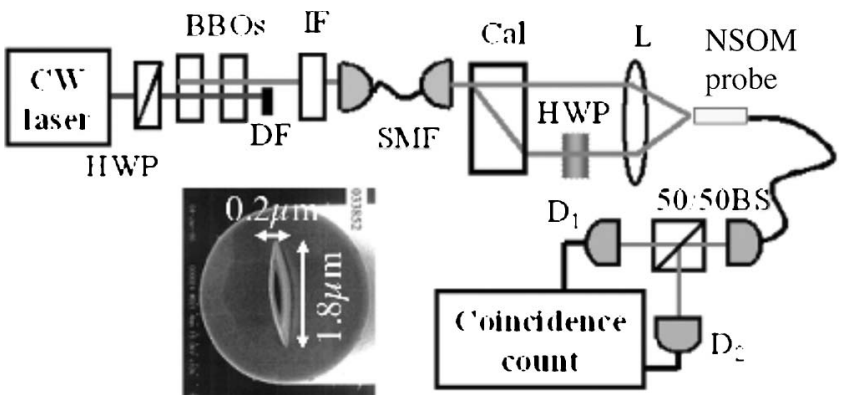

Fig. 6. Schematic of experimental setup. Photon pairs entangled in polarization are generated from two beta barium borate crystals (BBOs) and passed through an interference filter (IF) and a singlemode fiber (SMF). Then, the entangled photons are spatially separated into two paths depending on their polarization in a calcite crystal (Cal). The polarization in one path is rotated by $90^{\circ}$ by using a half-wave plate (HWP), giving a two-photon NOON state. An aspheric lens $(L)$ is used to focus the photons to a small spot and form an interference fringe in the focal plane. An NSOM probe with an elliptical opening (inset) is scanned with a piezoactuator along the focal plane. The SMF output of the probe is divided by a 50\% beam splitter (50/50 BS) and detected by two SPCMs. states of $|H V\rangle$ and $|V H\rangle$ propagate in a common path and cannot be discriminated (see Appendix A).

In the experiment, we adjusted the experimental setup to maximize the fidelity of the two-photon state $\rho_{s}$ to the ideal state. The real and imaginary parts of the density matrix of the state used in the previous experiments [15] are shown in Figs. 8(a) and 8(b). The fidelity of the polarization entangled state was high, $\overline{0.94}$, for an ideal state with $\xi=-1.27 \mathrm{rad}$ in Eq. (1). This state is almost a pure superposition of the $|2 H\rangle$ and $|2 V\rangle$ states because only the $|2 H\rangle\langle 2 H|| 2 H\rangle,\langle 2 V|$, $|2 V\rangle\langle 2 H|$, and $|2 V\rangle\langle 2 V|$ components are significant. We suppose that the residual error is not due to a Werner state (a statistical mixture of $|2 H\rangle,|2 V\rangle$, and $|H V\rangle$ ) as the $|H V\rangle\langle H V|$ component is almost negligible (see Figure 3), but rather due the extra $|H V\rangle$ components owing to the imperfect alignment or the birefringence control in the experimental setup of the photon pair source. Using this $\rho_{s}$, we calculated a two-photon interference fringe from Eq. (9) assuming plane-wave propagation, as shown in Fig. 8(c). The calculated interference fringe with a visibility of $94 \%$ oscillates with the reduced period of $\pi$. In Fig. 8(c), we note that the horizontal displacement of the fringe will vary substantially depending on an unknown bias phase in the interferometer, which should be a fitting parameter for comparison with the experimental data.

From this result and Eqs. (11) and (12), we calculated the two-photon interference fringe on the detection plane observed by the NSOM probe in order to compare it with the experimental data [squares in Fig. 8(d)]. First, the Gaussian envelope of the single-photon interference fringe was measured, and we determined the beam width at $1 / e^{2}$ to be $4.14 \mu \mathrm{m}$ (variance: $\sigma^{2}=1.07 \mu \mathrm{m}^{2}$ ). Then, because the Gaussian profile of the entangled photon pairs has half the width of that for a single-photon interference fringe [25], the $\sigma$ in Eq. (11) was set to be $\sigma / 2$. The intersecting angle $\theta$ was also experimentally estimated from the beam spacing after the calcite crystal and the focal length of the aspheric lens to be about $34^{\circ}$. Using Eq. (3), the lateral axis was converted from the phase $\phi$ to the position $r$ by using the relationship $r=\phi /(2 k \sin \theta)$. In addition, the aperture size of the NSOM probe was evaluated from SEM observations and was determined to be about $1.8 \times 10^{-6}$ and $2.0 \times 10^{-7} \mathrm{~m}$ in the long and short axes, respectively. Because the interference fringe was generated only in the direction of the short axis and the aperture had an ellipsoidal form, we geometrically determined the average aperture size $d=0.1 \mu \mathrm{m}$. Although these parameters were experimentally determined, we observed that the accuracy of the estimated values for the intersecting angle and

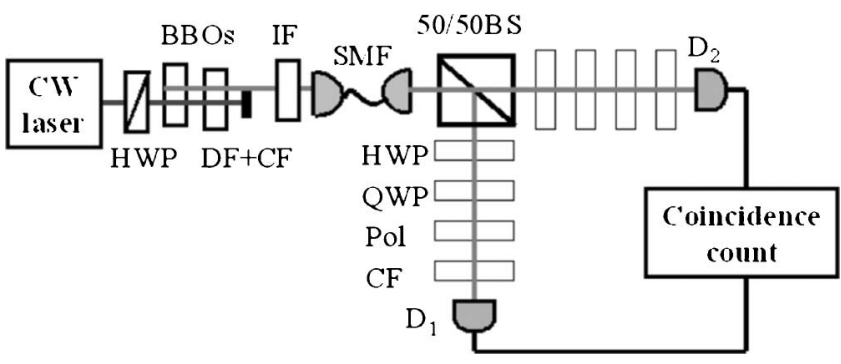

Fig. 7. Optical setup for the quantum state tomography measurement of the incident polarization entangled state: HWP, half-wave plate; QWP, quarter-wave plate; DF, dichroic filter; CF, color filter; IF, interference filter; SMF, single-mode fiber; Pol, polarizer; 50/50BS, 50\% beam splitter; and D1, 2, SPCM. 
aperture size were not sufficiently high, and we included errors of about $\pm 5^{\circ}$ and $\pm 0.05 \mu \mathrm{m}$. Using these experimentally obtained parameters and Eq. (12), we fitted the calculated two-photon interference fringe to the experimental data, as shown in Fig. 8(d). In this fitting procedure, we used only three fitting parameters: the maximum counting rate $(a)$, the center position $\left(r_{0}\right)$ of the Gaussian distribution, and an unknown bias phase in the interferometer. These initial values for the fitting were also estimated from the experimental data as $a=8 \mathrm{cps}, r_{0}=0.75 \mu \mathrm{m}$, and $0 \mathrm{rad}$. After the fitting, the fitting parameters were determined to be $7.6 \mathrm{cps}, 0.73 \mu \mathrm{m}$, and $-0.27 \mathrm{rad}$, respectively. We can see from Fig. 8(d) that the obtained curve fits well with almost all of the experimental data points. Particularly, each peak at around 0.2, 0.5, 0.8, 1.2, and $1.5 \mu \mathrm{m}$ and each valley at around $0.0,0.4,0.7,1.0$, and $1.3 \mu \mathrm{m}$ of the experimental data are well reproduced. This fact supports the validity of the analysis that has been proposed in this paper.

Based on this analysis, we can qualitatively discuss the cause of the degradation in the visibility. The main cause of the degradation in the obtained fringe is found to be the limited aperture size of the two-photon detector. If we had been able to use an efficient two-photon absorbing photosen-

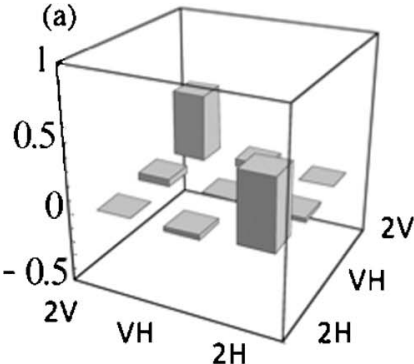

(c)

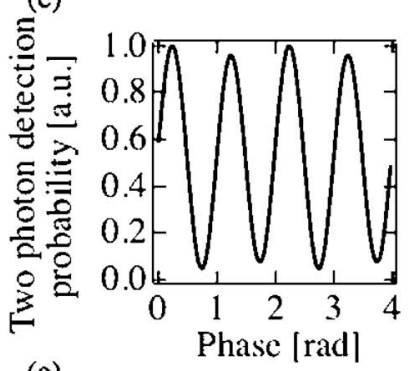

(e)

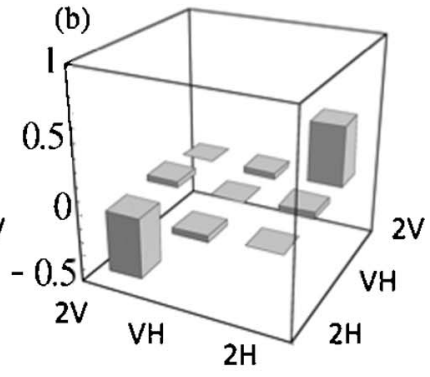

(d)

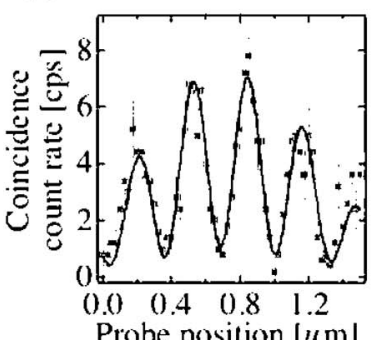

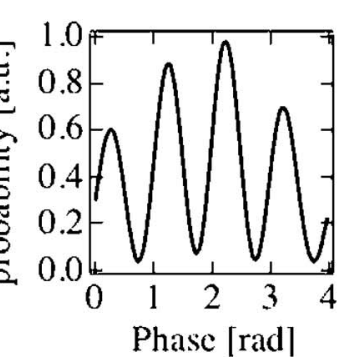

Fig. 8. Experimentally obtained density matrix [(a) real and (b) imaginary parts] after compensation. (c) Calculated two-photon interference fringe from Eq. (9) using density matrices (a) and (b). (d) Experimentally obtained two-photon interference fringe using polarization entangled state. The accumulation time for one data point was $5 \mathrm{~s}$, and the error bars show $\pm \sqrt{\text { counts }}$ assuming Poisson statistics. The solid curve indicates the fitting function of Eq. (12). (e) Calculated two-photon interference fringe from Eq. (11) using the experimentally obtained density matrix. sitive material in the current experimental setup, we could have observed a fringe with higher visibility of $94 \%$, as shown in Fig. 8(e), which shows the two-photon interference fringe on the detection plane without the limited aperture calculated by Eq. (11). For further improving the visibility, we need to consider the imperfections in the experimental setup. As shown in Fig. 8(d), the calculated interference fringe from Eq. (12) coincided well with the experimental data despite ignoring the imperfection of the interferometer in the calculation. As this fact suggests that the interferometer was very stable in the experiments, we only need to improve $\rho_{s}$ in order to further improve the quality of the interference fringe. From the above discussion of the density matrix, we can say that the degradation $\rho_{s}$ of the polarization entangled state was because of imperfect alignment or birefringence in the experimental setup. Therefore, by adjusting this imperfect alignment or birefringence in the experimental setup, the visibility of the interference fringe can be improved.

\section{CONCLUSION}

We proposed a method to simulate a two-photon interference fringe from an arbitrary density matrix as an input state. We discussed the influence of the degradation of the quality of the density matrix on the obtained interference fringe patterns (visibility and period) for various imperfections, such as loss and dephasing. Applying this method to the experimental data [15], we succeeded in reproducing the two-photon interference fringe from the experimentally obtained $\rho_{s}$ in which we used only three fitting parameters: the center position of the beam profile, the maximum intensity, and an unknown bias phase in the interferometer. Because the main cause of degradation of the interference fringe was found to be the limited aperture size of the NSOM probe, an interference fringe with a visibility of up to $94 \%$ can be observed if we can use an efficient two-photon absorbing material or a two-photon detector with a sufficiently high spatial resolution. In addition, from the discussion of the obtained density matrix, because the visibility limitation was attributed to imperfect alignment or birefringence control in the experimental setup, the visibility can be further improved by optimizing the experimental setup. Thus, for simulating a two-photon interference fringe with a high visibility and a period smaller than $\lambda / 2$, we conclude that the improvement of the imperfections in the polarization entangled state, such as the purification of the state (suppress the mixed state), the maximization of the entanglement, and the suppression of the polarization rotation in the optics, is indispensable, as is the use of an ultrastable interferometer and a two-photon detector with a high spatial resolution. Because we can use this method to examine the effect of the quality of an input entangled state on quantum interference, we believe that our proposed method can contribute toward further understanding the entangled state of the photons, not only for quantum lithography but also for quantum optics and communications.

\section{APPENDIX A: TRANSFORMATION OF THE $4 \times 4$ DENSITY MATRIX TO A $3 \times 3$ DENSITY MATRIX}

In Section $\underline{4}$, because the generated polarization entangled photons were passed through a SMF, we assumed that these photons were a monochromatic plane wave in single-mode 
propagation. Therefore, because the two-photon states of $|H V\rangle$ and $|V H\rangle$ propagate on a common path and cannot be discriminated, the obtained density matrix $\rho_{s}$ could be converted into a $3 \times 3$ matrix. According to Ref. [24], the $4 \times 4$ density matrix is described as

$$
\begin{aligned}
\rho_{4 \times 4}= & A_{11}|V V\rangle\left\langle V V\left|+A_{12}\right| V V\right\rangle\left\langle V H\left|+A_{13}\right| V V\right\rangle\langle H V| \\
& +A_{14}|V V\rangle\left\langle H H\left|+A_{12}^{*}\right| V H\right\rangle\left\langle V V\left|+A_{22}\right| V H\right\rangle\langle V H| \\
& +A_{23}|V H\rangle\left\langle H V\left|+A_{24}\right| V H\right\rangle\left\langle H H\left|+A_{13}^{*}\right| H V\right\rangle\langle V V| \\
& +A_{23}^{*}|H V\rangle\left\langle V H\left|+A_{33}\right| H V\right\rangle\left\langle H V\left|+A_{34}\right| H V\right\rangle\langle H H| \\
& +A_{14}^{*}|H H\rangle\left\langle V V\left|+A_{24}^{*}\right| H H\right\rangle\left\langle V H\left|+A_{34}\right| H H\right\rangle\langle H V| \\
& +\left(1-A_{11}-A_{22}-A_{33}\right)|H H\rangle\langle H H| .
\end{aligned}
$$

On the other hand, the $3 \times 3$ density matrix can be defined as

$$
\begin{aligned}
\rho_{3 \times 3}= & B_{11}|V V\rangle\left\langle V V\left|+B_{12}\right| V V\right\rangle\left\langle V H\left|+B_{13}\right| V V\right\rangle\langle H H| \\
& +B_{12}^{*}|V H\rangle\left\langle V V\left|+B_{22}\right| V H\right\rangle\left\langle V H\left|+B_{23}\right| V H\right\rangle\langle H H| \\
& +B_{13}^{*}|H H\rangle\left\langle V V\left|+B_{23}^{*}\right| H H\right\rangle\langle V H| \\
& +\left(1-B_{11}-B_{22}\right)|H H\rangle\langle H H| .
\end{aligned}
$$

From these density matrices, we attempted to obtain the relationships between $A_{i j}$ and $B_{i j}$. In the experimental setup shown in Fig. 6, the incident photons were separated using a 50/50 BS for the coincidence measurement. Assuming the backpropagation of the photons from a detector to a crystal via a 50/50 BS, we consider the relationships of these coefficients. The 50/50 BS can be described as

$$
\left(\begin{array}{l}
\hat{a}_{\gamma} \\
\hat{a}_{\delta}
\end{array}\right)=\frac{1}{\sqrt{2}}\left(\begin{array}{cc}
1 & 1 \\
-1 & 1
\end{array}\right)\left(\begin{array}{l}
\hat{a}_{\alpha} \\
\hat{a}_{\beta}
\end{array}\right),
$$

where $\hat{a}_{i}(i=\alpha, \beta, \gamma, \delta)$ is the annihilation operator. The suffixes $\alpha$ and $\beta$ indicate the two input ports from the detectors to the $50 / 50 \mathrm{BS}$, and the suffixes $\gamma$ and $\delta$ indicate the output ports from the 50/50 BS to the crystal and free space, respectively. Using this relationship, we consider the component $A_{11}|V V\rangle\langle V V|$ as an example. This component can be described as follows for the coincidence counts by the two detectors in the ports $\alpha$ and $\beta$.

$$
A_{11}|V V\rangle\left\langle V V\left|=A_{11}\right| V\right\rangle_{\beta}|V\rangle_{\alpha}\left\langleV | _ { \alpha } \left\langle\left. V\right|_{\beta}\right.\right.
$$

Using the relationship in Eq. (A3), we modified this component as

$$
A_{11}|V V\rangle\left\langleV V \left|=\frac{A_{11}}{2}\left(|V V\rangle_{\gamma}-|V V\rangle_{\delta}\right)\left(-\left\langle\left. V V\right|_{\delta}+\left\langle\left. V V\right|_{\gamma}\right) .\right.\right.\right.\right.
$$

Because no photons come from the $\delta$ port, this equation can be written as

$$
A_{11}|V V\rangle\langle V V|=\frac{A_{11}}{2}\left(|V V\rangle_{\gamma}\left\langle\left. V V\right|_{\gamma}\right) .\right.
$$

Then, by comparing this equation with Eq. (A2), we finally obtained the relationship between $B_{11}$ and $A_{11}$ :

$$
B_{11}=\frac{A_{11}}{2}
$$

Similarly, we compared the other coefficients and obtained their relationships as follows:

$$
\begin{aligned}
& B_{12}=\frac{1}{2 \sqrt{2}} \frac{A_{12}+A_{13}}{2}, \\
& B_{13}=\frac{A_{14}}{2}, \\
& B_{22}=\frac{1}{4} \frac{A_{22}+A_{23}+A_{33}}{3}, \\
& B_{23}=\frac{1}{2 \sqrt{2}} \frac{A_{24}+A_{34}}{2} .
\end{aligned}
$$

Then, by taking $\rho_{3 \times 3} / \operatorname{Tr}\left[\rho_{3 \times 3}\right]$, we finally obtained $\rho_{3 \times 3}$. Thus, after experimentally determining the coefficients $A_{i j}$ of $\rho_{4 \times 4}$ from the projection measurements, we converted the obtained $4 \times 4$ density matrix to a $3 \times 3$ density matrix.

\section{ACKNOWLEDGMENTS}

The authors thank H. F. Holger and T. Nagata for their technical suggestions. This work was supported by the Japan Science and Technology Agency (JST), the Ministry of Internal Affairs and Communication, the Japan Society for the Promotion of Science, the 21st Century COE Program, and the Special Coordination Funds for Promoting Science and Technology.

\section{REFERENCES}

1. A. N. Boto, P. Kok, D. S. Abrams, S. L. Braunstein, C. P. Williams, and J. P. Dowling, "Quantum interferometric optical lithography: exploiting entanglement to beat the diffraction limit," Phys. Rev. Lett. 85, 2733-2736 (2000).

2. P. Kok, A. N. Boto, D. S. Abrams, C. P. Williams, S. L. Braunstein, and J. P. Dowling, "Quantum-interferometric optical lithography: towards arbitrary two-dimensional patterns," Phys. Rev. A 63, 063407 (2001)

3. G. Bjork and L. L. Sanchez-Soto, "Entangled-state lithography: tailoring any pattern with a single state," Phys. Rev. Lett. 86, 4516-4519 (2001).

4. M. D'Angelo, M. V. Chekhova, and Y. Shih, "Two-photon diffraction and quantum lithography," Phys. Rev. Lett. 87, 013602 (2001).

5. T. Nagata, R. Okamoto, J. L. O’Brien, K. Sasaki, and S. Takeuchi, "Beating the standard quantum limit with four entangled photons," Science 316, 726-729 (2007).

6. K. Edamatsu, R. Shimizu, and T. Itoh, "Measurement of the photonic de Broglie wavelength of entangled photon pairs generated by spontaneous parametric down-conversion," Phys. Rev. Lett. 89, 213601 (2002).

7. M. W. Mitchell, J. S. Lundeen, and A. M. Steinberg, "Super-resolving phase measurements with a multiphoton entangled state," Nature 429, 161-164 (2004).

8. P. Walther, J.-W. Pan, M. Aspelmeyer, R. Ursin, S. Gasparoni, and A. Zeilinger, "De Broglie wavelength of a non-local fourphoton state," Nature 429, 158-161 (2004).

9. F. W. Sun, B. H. Liu, Y. F. Huang, Z. Y. Ou, and G. C. Guo, "Observation of the four-photon de Broglie wavelength by stateprojection measurement," Phys. Rev. A 74, 033812 (2006).

10. B. H. Liu, F. W. Sun, Y. X. Gong, Y. F. Huang, G. C. Guo, and Z. Y. Ou, "Four-photon interference with asymmetric beam splitters," Opt. Lett. 32, 1320-1322 (2007).

11. K. J. Resch, K. L. Pregnell, R. Prevedel, A. Gilchrist, G. J. Pryde, J. L. O'Brien, and A. G. White, "Time-reversal and superresolving phase measurements," Phys. Rev. Lett. 98, 223601 (2007). 
12. J. G. Rarity, P. R. Tapster, E. Jakeman, T. Larchuk, R. A. Campos, M. C. Teich, and B. E. A. Saleh, "Two-photon interference in a Mach-Zehnder interferometer," Phys. Rev. Lett. 65, 1348-1351 (1990).

13. E. J. S. Fonseca, C. H. Monken, and S. Pauda, "Measurement of the de Broglie wavelength of a multiphoton wave packet," Phys. Rev. Lett. 82, 2868-2871 (1999).

14. H. S. Eisenberg, J. F. Hodelin, G. Khoury, and D. Bouwmeester, "Multiphoton path entanglement by nonlocal bunching," Phys. Rev. Lett. 94, 090502 (2005).

15. Y. Kawabe, H. Fujiwara, R. Okamoto, K. Sasaki, and S. Takeuchi, "Quantum interference fringes beating the diffraction limit," Opt. Exp. 15, 14244-14250 (2007).

16. Y. H. Kim, S. P. Kulik, and Y. Shih, "High-intensity pulsed source of space-time and polarization double-entangled photon pairs," Phys. Rev. A 62, 011802 (2000).

17. C. K. Hong, Z. Y. Ou, and L. Mandel, "Measurement of subpicosecond time intervals between two photons by interference," Phys. Rev. Lett. 59, 2044-2046 (1987).

18. C. C. Gerry and R. A. Campos, "Generation of maximally entangled photonic states with a quantum-optical Fredkin gate," Phys. Rev. A 64, 063814 (2001).
19. J. L. O'Brien, G. J. Pryde, A. G. White, T. C. Ralph, and D. Branning, "Demonstration of an all-optical quantum controlled-NOT gate," Nature 426, 264-267 (2003).

20. E. M. Nagasako, S. J. Bentley, R. W. Boyd, and G. S. Agarwal, "Nonclassical two-photon interferometry and lithography with high-gain parametric amplifiers," Phys. Rev. A 64, 043802 (2001).

21. Y.-H. Wang and L.-M. Kuang, "Nonmaximally entangled state quantum photolithography,” J. Opt. B: Quantum Semiclass. Opt. 5, 405-408 (2003).

22. R. F. Werner, "Quantum states with Einstein-Podolsky-Rosen correlations admitting a hidden-variable model," Phys. Rev. A 40, 4277-4281 (1989).

23. E. Betzig, J. K. Trautman, T. D. Harris, J. S. Weiner, and R. L. Kostelak, "Breaking the diffraction barrier: optical microscopy of a nanometric scale," Science 251, 1468-1470 (1991).

24. D. F. V. James, P. G. Kwiat, W. J. Munro, and A. G. White, "Measurement of qubits," Phys. Rev. A 64, 052312 (2001).

25. M. B. Nasr, A. F. Abouraddy, M. C. Booth, B. E. A. Saleh A. V. Sergienko, M. C. Teich, M. Kempe, and R. Wolleschensky, "Biphoton focusing for two-photon excitation," Phys. Rev. A 65, 023816 (2002). 